\title{
SATURATION EFFECTS OF DARK MAGNETO-OPTICAL RESONANCES OBSERVED ON THE ALKALI $D_{l}$ LINE
}

\author{
L. Kalvans \\ Laser Centre, University of Latvia, \\ 19 Rainis Blvd., LV-1586, Riga, LATVIA \\ e-mail: linards.kalvans@1u.lv
}

\begin{abstract}
Theoretical studies of saturation effects of dark magneto-optical resonances are presented. A model based on the optical Bloch equations is applied to simulate numerically the alkali $D_{l}$ excitation in external magnetic field. The laser power densities at which the expected dark resonance contrast reaches maximum are analyzed and correlated with the quantum superposition states induced by exciting laser field. Fictional hyperfine structure constants for the excited state are used to vary the selection efficiency of hyperfine transitions.
\end{abstract}

Key words: magneto-optical resonance, dark state.

\section{INTRODUCTION}

The phenomenon of coherent population trapping (CPT) has attracted a steady interest in recent years. The effect has a number of possible applications in the high-precision magnetometry [1], laser cooling [2], atomic clocks [3], ultraslow group velocity propagation of light [4], and others. One way of exploring the CPT involves magneto-optical resonances which are observed when a fluorescence signal is registered as a function of magnetic field. If there is a minimum in the fluorescence signal at zero magnetic field the resonance is called dark, and in the opposite case - bright. Dark resonances have been observed both for wide and narrow spectral line excitation $[5,6]$, while the bright ones have been reported only in the latter case [7]. Of course, the case of narrow exciting line-width is of more practical interest, as in this situation atoms are prepared in a particular quantum state which can be described in detail theoretically or anticipated from experimental results. A common physical tool to investigate the resonances is $D_{1}$ line of alkali metals (Fig. 1) in atomic vapour. If the resonances are observed in $\mathrm{cm}$-sized vapour cell, which is the usual case, distinct hyperfine transitions are resolved only for Caesium and partially resolved for ${ }^{87} \mathrm{Rb}$, while in the cases of ${ }^{85} \mathrm{Rb}$ and more lightweight alkali metals the hyperfine transitions are covered by Doppler broadening. Despite that, by using a laser with a sufficiently narrow spectral profile the expected resonances can be observed and described theoretically also in the presence of the Doppler broadening [8].

The resonances are characterized by the depth (or contrast) and the width, both being dependent on the power density of the laser inducing the fluorescence. Both attributes depend also on other experimental parameters - such as the laser beam's diameter and the temperature in the cell, but in the scope of this paper it will be always assumed that these experimental parameters are constant for all 
cases considered. The width of resonance is always increasing as the laser power density increases; concerning the contrast, the dependence is not as straightforward for this parameter, since the contrast reaches maximum at some power density and decreases if the latter is increasing further.

In many cases, saturation of the dark resonance at higher laser power densities has been observed $[9,10]$, though there is very little research done to understand in detail the physical processes that govern the saturation. Thus the goal of this research is to find correlation between the contrast saturation and the number of atoms being prepared in a particular quantum state responsible for the resonance formation, and the dependence of both quantities on the selectivity of excitation for particular hyperfine transitions in $D_{l}$ line.

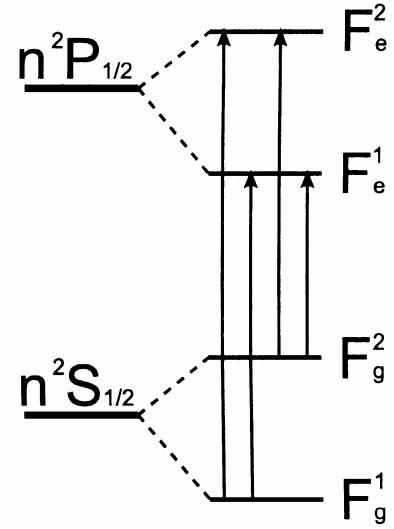

Fig. 1. Schematic structure of the alkali $D_{l}$ line; vertical arrows represent four distinct hyperfine transitions, and $\mathrm{F}_{\mathrm{g}}^{1}=\mathrm{F}_{\mathrm{e}}^{1}, \mathrm{~F}_{\mathrm{g}}{ }^{2}=\mathrm{F}_{\mathrm{e}}^{2}$ are quantum numbers for the total atomic angular momentum.

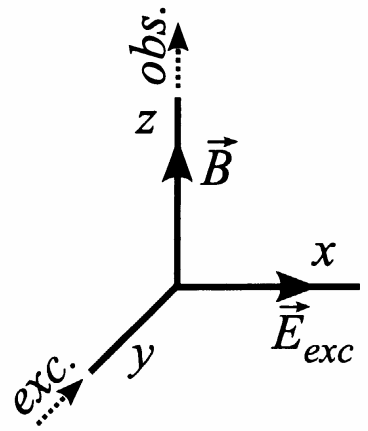

Fig. 2. Excitation and observation geometry for dark resonance investigation

\section{THEORY}

The research methods of this paper include numerical calculations based on theoretical model describing the atom-laser interaction in the dipole approximation. The excitation in all cases is supposed to be linearly polarized with the electric field vector oscillating perpendicularly to the applied magnetic field, while the fluorescence is observed in the same direction as magnetic field is applied (see Fig. 2). The propagation direction of laser beam is insignificant in the case of theoretical research presented here as long as the above conditions are maintained; however, in experiments the latter is usually chosen to be perpendicular to the observation direction to minimize the background of scattered light in the fluorescence signal. For the excitation conditions defined above there are two nonzero spherical components of the oscillating electric field, namely $\mathrm{e}^{+1}$ and $\mathrm{e}^{-1}$ if the quantization axis is chosen along the B-field, which is a logical choice. Those two components act on the atomic system inducing two-photon couplings which form so-called dark and bright states. The dark state is formed when the two of the ground state magnetic sublevels are coupled via one excited state sublevel (Fig. 3) 
forming a quantum superposition state that cannot absorb light and therefore cannot produce fluorescence. The bright state is formed when two excited state sublevels are coupled via one ground state sublevel and the atom's ability to absorb light and emit it as fluorescence is not affected (herefrom the name "bright state"). When all the magnetic sublevels are degenerate (at zero magnetic field) the twophoton resonance condition holds true (for the chosen hyperfine transition) and both superpositions are formed. The magnetic field shifts the magnetic sublevels, destroying the conditions for two-photon resonance. If the total atomic angular momentum of the ground state is greater than or equal to that of excited state $\left(\mathrm{F}_{\mathrm{g}} \geq\right.$ $F_{e}$ ), the dark states are dominant at the zero field, therefore the fluorescence increases when the field is applied and a dark resonance is observed. The situation is opposite if the angular momentum is larger for excited state $\left(\mathrm{F}_{\mathrm{g}}<\mathrm{F}_{\mathrm{e}}\right)$ and bright resonance is observed. Thus three of four hyperfine transitions in a D1 line meet conditions for formation of a dark resonance and are subject to our investigation.

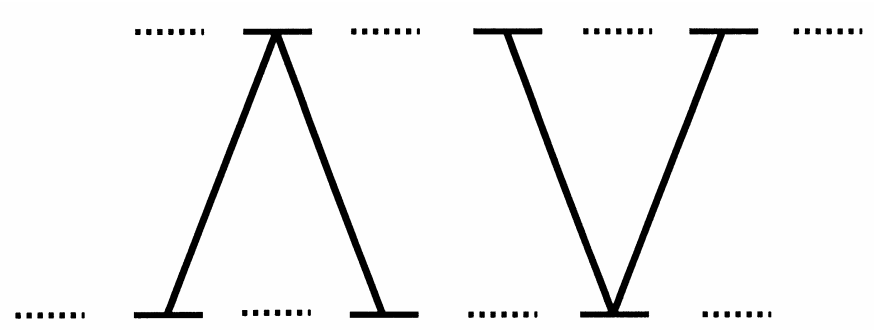

Fig. 3. Formation of dark and bright quantum superposition states. Horizontal lines indicate magnetic sublevels of ground and excited states. Left-hand side: the laser field couples two ground-state magnetic sublevels via one sublevel of the excited state, no light is absorbed or emitted - a dark state is formed. Right-hand side: two excited state sublevels are coupled with one of the ground state, light is absorbed and emitted - a bright state is formed.

Detailed theoretical description is based on the optical Bloch equations (OBEs) [11] which describe the time evolution of semi-classical quantum density matrix:

$$
i \hbar \frac{\partial \rho}{\partial t}=[\hat{H}, \rho]+i \hbar \hat{R} \rho
$$

where $\hat{R}$ represents the relaxation operator and $\hat{H}=\hat{H}_{0}+\hat{H}_{B}+\hat{V}$ is the full Hamiltonian of the atom that interacts with laser light in the presence of $d c$ magnetic field. With $\hat{H}_{0}$ being the unperturbed Hamiltonian of an atom depending on its internal coordinates, $\hat{H}_{B}$ is the Hamiltonian of atomic interaction with magnetic field, and $\hat{V}=-\hat{\vec{d}} \cdot \vec{E}(t)$ is the interaction operator in the dipole approximation, where $\hat{\vec{d}}$ is the electric dipole operator and $\vec{E}(t)$ - the electric field of the excitation radiation. The light is treated classically and assumed to possess a fluctuating phase giving it a finite line-width $\Delta \omega$ and a power distribution with a Lorentzian profile. 
The dipole operator $\hat{\vec{d}}$ consists of the following matrix elements coupling levels $|i\rangle$ and $|j\rangle: d_{1}^{i j}=\langle i|\vec{d} \cdot \vec{e}| j\rangle$. Each level $|i\rangle$ is described by the total atomic angular momentum $F$ and the magnetic quantum number $M$; when the magnetic field is applied the hyperfine levels represented by $F$ are mixed within ground or excited state.

The OBEs are expanded when the rotating wave approximation [12] is applied and adiabatic elimination is performed excluding the optical coherences and obtaining the following rate equations for Zeeman's coherences:

$$
\begin{aligned}
& \frac{\partial \rho_{g_{i} g_{j}}}{\partial t}=\left(\Gamma_{p, g_{i} e_{m}}+\Gamma_{p, e_{k} g_{j}}^{*}\right) \sum_{e_{k}, e_{m}}\left(d_{1}^{g_{i} e_{k}}\right)^{*} d_{1}^{e_{m} g_{j}} \rho_{e_{k} e_{m}}- \\
& -\sum_{e_{k}, g_{m}}\left[\Gamma_{p, e_{k} g_{j}}^{*}\left(d_{1}^{g_{i} e_{k}}\right)^{*} d_{1}^{e_{k} g_{m}} \rho_{g_{m} g_{j}}+\Gamma_{p, g_{i} e_{k}}\left(d_{1}^{g_{m} e_{k}}\right)^{*} d_{1}^{e_{k} g_{j}} \rho_{g_{i} g_{m}}\right]- \\
& -i \omega_{g_{i} g_{j}} \rho_{g_{i} g_{j}}+\sum_{e_{k}, e_{m}} \Gamma_{g_{i} g_{j}}^{e_{k} e_{m}} \rho_{e_{k} e_{m}}-\gamma \rho_{g_{i} g_{j}}+\lambda \delta\left(g_{i}, g_{j}\right) \rho_{g_{i} g_{j}} ; \\
& \frac{\partial \rho_{e_{i} e_{j}}}{\partial t}=\left(\Gamma_{p, e_{i} g_{m}}^{*}+\Gamma_{p, g_{k} e_{j}}\right) \sum_{g_{k}, g_{m}} d_{1}^{e_{i} g_{k}}\left(d_{1}^{g_{m} e_{j}}\right)^{*} \rho_{g_{k} g_{m}}- \\
& -\sum_{g_{k}, e_{m}}\left[\Gamma_{p, g_{k} e_{j}} d_{1}^{e_{i} g_{k}}\left(d_{1}^{g_{m} e_{j}}\right)^{*} \rho_{e_{m} e_{j}}+\Gamma_{p, e_{i} g_{k}}^{*} d_{1}^{e_{m} g_{k}}\left(d_{1}^{g_{k} e_{j}}\right)^{*} \rho_{e_{i} e_{m}}\right]- \\
& -i \omega_{e_{i} e_{j}} \rho_{e_{i} e_{j}}-\Gamma \rho_{g_{i} g_{j}} \text {. }
\end{aligned}
$$

In Eqs. (2) and (3), $\rho_{g_{i} g_{j}}$ and $\rho_{e_{i} e_{j}}$ denote the density matrices for the ground and excited state, $\Gamma_{p, g_{i} e_{j}}$ is the interaction strength between levels $\left|g_{i}\right\rangle$ and $\left|e_{j}\right\rangle$ which depends on the Rabi frequency, the natural line-width of the excited state, the laser line-width and laser detuning away from the transition between states $\left|g_{i}\right\rangle$ and $\left|e_{j}\right\rangle$. All terms in the both equations can be physically interpreted - the first term on the right-hand side of Eq. (2) represents the rate at which the population is driven by laser field from the excited state to the ground state, while the second denotes the opposite process. The third term describes destruction of Zeeman's coherences in the ground state due to the shifting of magnetic sublevels in the magnetic field and can be calculated according to the Breit-Rabi formula; the fourth term stands for the coherence transfer from excited state via spontaneous transitions, and the fifth is the relaxation rate of ground state as atoms are leaving the interaction region due to thermal motion (transit relaxation) that can be calculated from the laser beam diameter and mean thermal velocity. The final term in Eq. (2) is the rate at which non-polarized atoms are supplied into the interaction region due to the thermal motion. It is assumed that the atomic equilibrium density outside the interaction region is normalized to unity, which leads to $\lambda=\gamma$.

In Eq. (3) the first term on the right-hand side describes the population transfer rate from ground to excited state induced by laser, and the second term denotes the induced population transfer in the opposite direction. The third term describes destruction of coherences due to energy shifts between different magnetic 
sublevels and the fourth refers to the relaxation of the excited state $\Gamma=\Gamma_{N}+\gamma$, with $\Gamma_{N}$ being the natural line-width and $\gamma$ - the transit relaxation rate described above.

These equations were successfully applied to describe experimental data of dark and bright resonances in Caesium [13] and Rubidium [8, 14] atomic vapour media; these articles and references therein contain a more detailed description of the theoretical model.

The equations are solved for steady-state conditions $\partial \rho / \partial t=0$, obtaining the atomic density matrices for the ground and excited state. The density matrices allow calculating the fluorescence signal, while the matrices themselves contain information about the states of the atoms. The (relative) number of atoms present in dark or bright states can be directly obtained from the density matrices. The characteristic relaxation times for the bright states (natural line-width) are roughly two orders of magnitude larger than those for the dark states (transit relaxation), so it is a good approximation to consider that only the behaviour of the dark states is responsible for the formation of dark resonances. The dark states can be formed on Zeeman's sublevels from each of ground state hyperfine levels as well as on their combination. For analysis only those dark states are used which involve Zeeman's sublevels solely from the hyperfine transition to which the laser frequency is tuned on. The rest of dark states are formed for already large two-photon detuning and are very little affected by the relatively small energy shifts caused by the magnetic field.

The latter assumption must be changed significantly when the Doppler effect is taken into account, as in this case atoms from different velocity groups "see" different wavelengths of the exciting light, and the atoms that are in resonance with other hyperfine transitions are present if the hyperfine energy splits are below the width of the Doppler profile. In order to broaden the scope of the research, a hypothetical atomic $D_{l}$ line is chosen for which the hyperfine constants can be adjusted as necessary thus changing the energy splits between hyperfine levels. Moreover, we would like to change only the hyperfine constant for the excited state as in real-life atoms the ground state $h f s$ splits are much larger than the Doppler broadening of spectral lines. In order to look at a somewhat simpler picture, a system of $D_{l}$ hyperfine states is chosen with the total atomic quantum number being equal to either 1 or 2 (see Fig. 1). The results can however be generalized for the lines with greater quantum numbers; in this way we would obtain fairly objective conditions to draw conclusions on how the hyperfine splits in the excited state affect the saturation of dark magneto-optical resonance. It is worth noting that such $D_{l}$ line configuration is present in $\mathrm{Na}$ and ${ }^{87} \mathrm{Rb}$ atoms.

Though for such simulations with fictional atomic constants exact values of theoretical parameters are not of so great importance, they are assumed as follows: the natural line-width of $D_{l}$ line $-6 \mathrm{MHz}$, the full width at half-maximum of the Doppler profile $-500 \mathrm{MHz}$, the transit relaxation rate $-0.03 \mathrm{MHz}$ and the linewidth of exciting laser - $10 \mathrm{MHz}$. All are typical values for alkali atoms excited at room temperature in a $\mathrm{cm}$-sized cell. To model different excitation laser power densities (I) the Rabi frequency $(\Omega)$ was altered, which is a common quantity used to characterize the strength of laser-induced coupling between distinct atomic states, since $I \sim \Omega^{2}$, the squared value of the Rabi frequency being used as a substitute for the laser power density in the data analysis. 


\section{RESULTS AND DISCUSSION}

We will start with analyzing the dependence of the resonance contrast and number of atoms on the squared Rabi frequency for various values of the excited state hyperfine splits $\left(\Delta_{h f s}\right)$. In order to emphasize the importance of Doppler broadening in observation of the magneto-optical resonances in atomic vapour, in the plots the ratio $\Delta_{h f s} / \mathrm{FWHM}_{\text {Doppler }}$ is shown. Figures $4 a-c$ show the dependence of the resonance contrast and atoms trapped in the dark state for the hyperfine transition $\mathrm{F}_{\mathrm{g}}=1 \rightarrow \mathrm{F}_{\mathrm{e}}=1$ (heretofore referred to as transition (1)), while Fig. $4 d$ shows the maxima positions for both quantities as a function of excited state hyperfine split. It can be clearly seen that in all cases the maxima in the resonance contrast are observed at lower values of $\Omega^{2}$ (which, as mentioned above, correspond to the laser power density), The relative distance between both starts shrinking as the $h f s$ splitting increases, which can be seen comparing Figs. $4 b$ and $4 c$ (note that the abscissa values are given in logarithmic scale) indicating that for very large $h f s$ splits both lines in Fig. $4 d$ would become parallel.
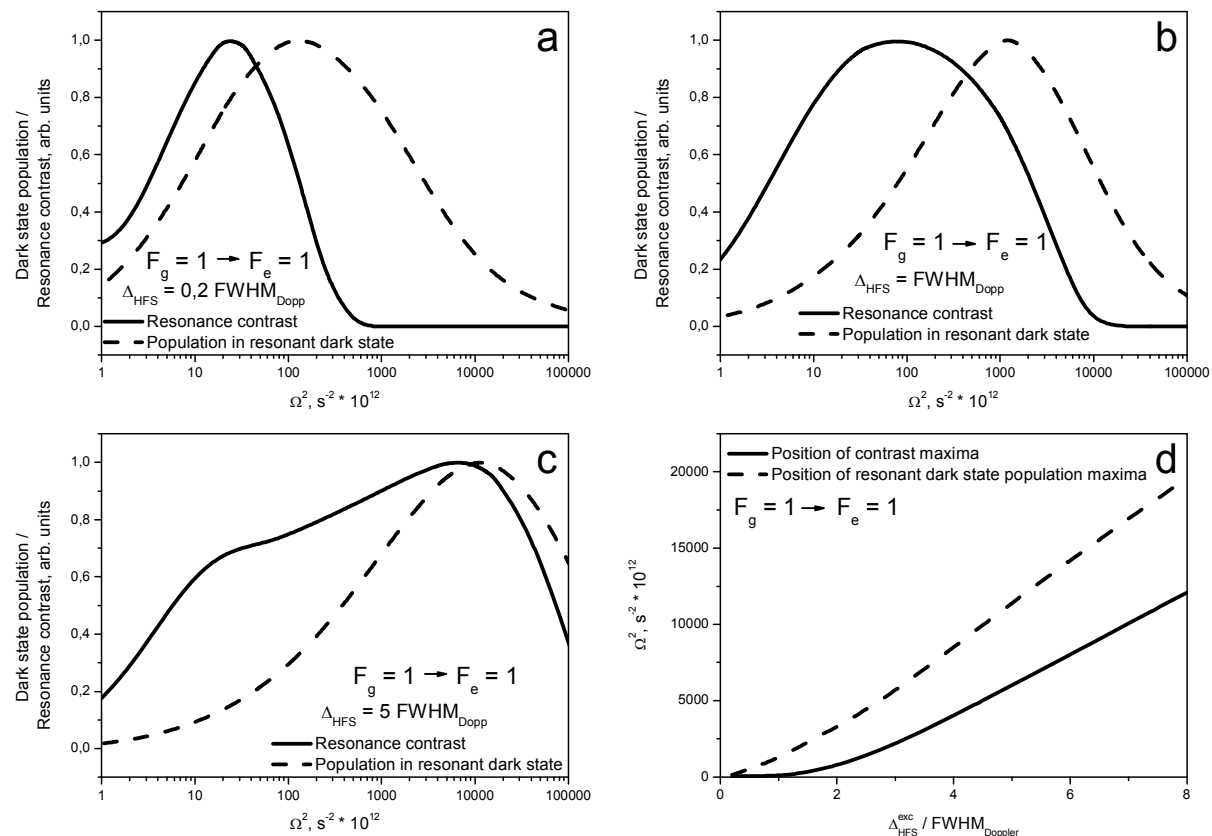

Fig. 4. $(a-c)$ Dependence of the resonance contrast (solid line) and the number of atoms in the resonant dark state (dashed line) on Rabi frequency squared for various hyperfine splits in the excited state for $\mathrm{F}_{\mathrm{g}}=1 \rightarrow \mathrm{F}_{\mathrm{e}}=1$ transition; $(d)$ position of maximums of the above values as a function of the hyperfine split in the excited state.

Figure 5, similarly to Fig. $4 a-c$, shows the dependence of the resonance contrast and the number of atoms in the resonant dark state on Rabi frequency squared for the hyperfine transition $F_{g}=2 \rightarrow F_{e}=1$ (transition (2)) for various excited state hyperfine splitting values. Here also, at smaller splitting the maxima in the resonance contrast "outpace" those of the dark state population, though the situation changes at larger splitting when the resonance maxima appear at lower 
laser power densities $\left(\Omega^{2}\right)$. In Fig. $5 d$, where the maxima positions are shown, both lines again tend to become parallel, but by that time the line depicting contrast is higher.
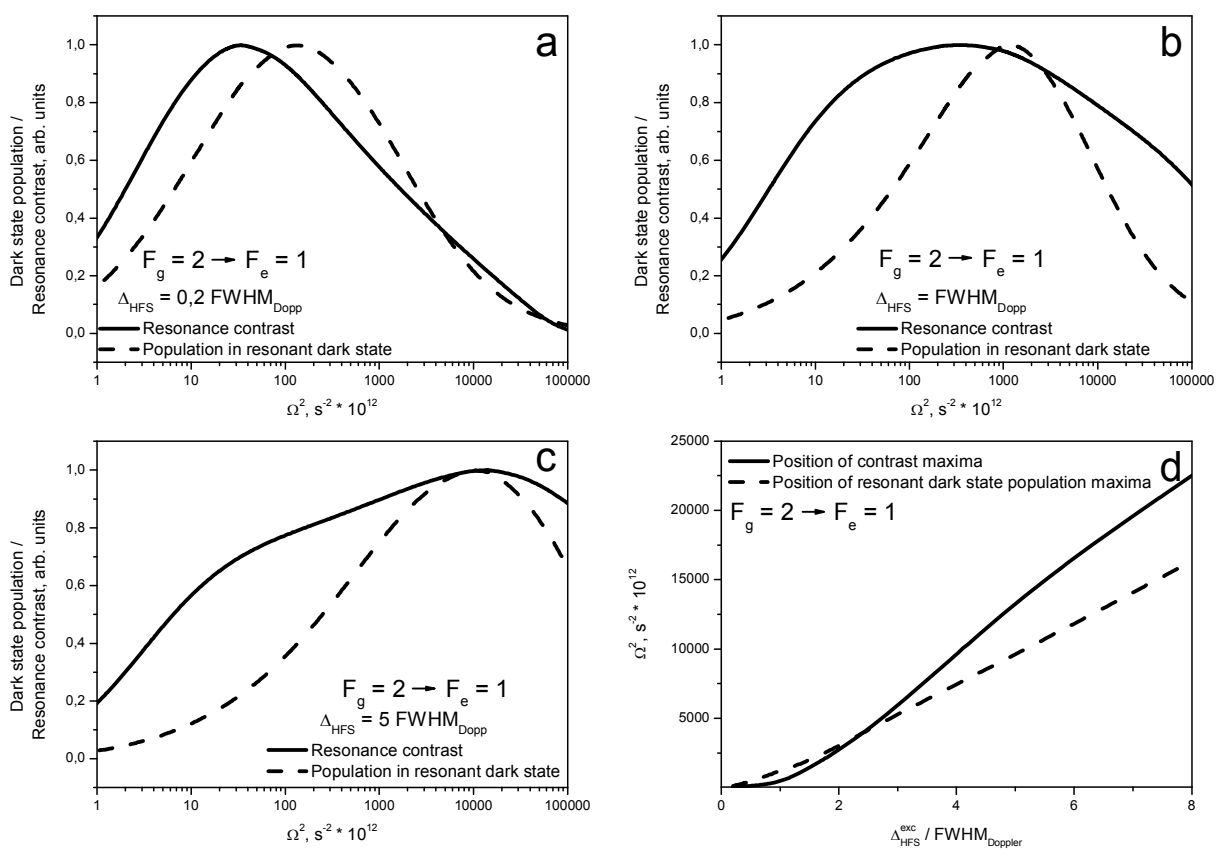

Fig. 5. $(a-c)$ Dependence of the resonance contrast (solid line) and the number of atoms in the resonant dark state (dashed line) on the Rabi frequency squared for various hyperfine splits in the excited state for $\mathrm{F}_{\mathrm{g}}=2 \rightarrow \mathrm{F}_{\mathrm{e}}=1$ transition; $(d)$ position of maximums of the above values as function of the hyperfine split in the excited state.

Concluding the set of power dependences is Fig. 6, which depicts the situation with the third hyperfine transition in the chosen $D_{l}$ scheme that is responsible for dark resonance $\left(\mathrm{F}_{\mathrm{g}}=2 \rightarrow \mathrm{F}_{\mathrm{e}}=2\right.$ - transition (3)). Here as well as in Fig. 4, at all $h f s$ splits the maxima of the resonance contrast are found to appear at lower laser power densities as the maxima of dark state population, and, once again, at larger splitting the shift between both maxima becomes nearly constant.

Absolute contrast maxima values for all three hyperfine transitions as a function of the hyperfine splitting are shown in Figs. $7 a-c$ with a solid line. It can be seen that for transitions (1) and (2) the maximum contrast increases for all split values, while for the third transition there is a local maximum when the ratio $\Delta_{h f s} / \mathrm{FWHM}_{\text {Doppler }}$ is close to zero. The latter can be explained by the fact that transition (3) is accompanied by transition (2), which produces dark resonances with the largest contrasts in the set of hyperfine transitions discussed here. When the energy difference between those two hyperfine transitions is small compared to the Doppler profile, both lines contribute to the formation of the dark resonance producing the mentioned maxima. As the energy difference increases, the role of the resonant transition becomes more important and the contrast produced by it becomes dominant. 

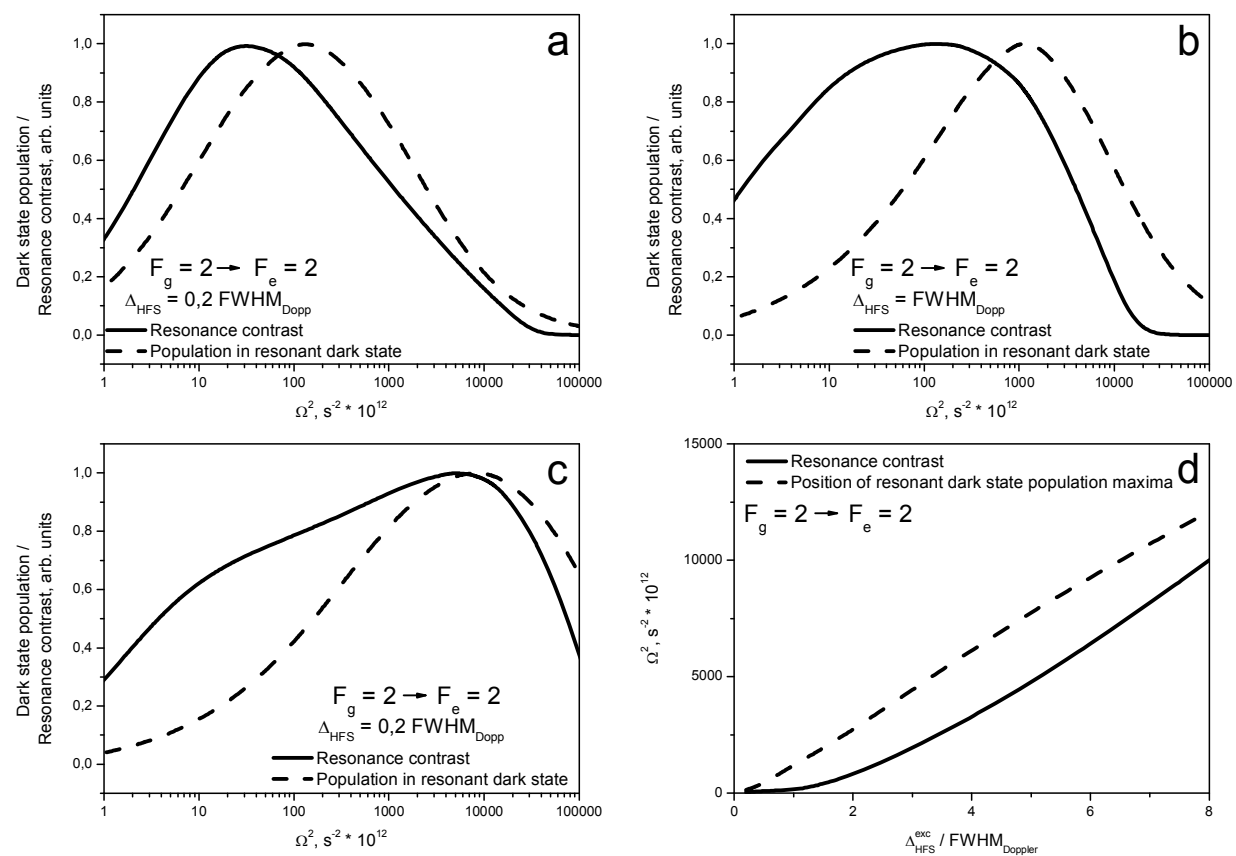

Fig. 6. $(a-c)$ Dependence of the resonance contrast (solid line) and the number of atoms in the resonant dark state (dashed line) on Rabi frequency squared for various hyperfine splits in the excited state for $\mathrm{F}_{\mathrm{g}}=2 \rightarrow \mathrm{F}_{\mathrm{e}}=2$ transition; $(d)$ position of maxima of the above values as a function of the hyperfine split in the excited state.

In Figs. $7 a-c$ the dashed lines show the number of atoms in the resonant dark states at the Rabi frequency for which the resonance maximum is predicted, while the dotted - the maximum values of the population for a given $h f s$ split (note that the vertical scale for these two values is different from that for the contrast). Both values correlate very well with the expected resonance contrast, especially the first one. In order to emphasize this, Fig. $7 d$ shows the ratio between the maximum contrast value and the dark-state population that corresponds to the given dark resonance. Both transitions originating at $\mathrm{F}_{\mathrm{g}}=2$ tend to have a very large value of this ratio when there is no splitting; this could be explained by the fact that at larger $h f s$ splits the contrast maximum is reached at higher Rabi frequencies. This means that in this case the optical pumping of atoms away from resonant states is more effective, so the atoms "released" from the dark state are pumped away from the resonant states and contribute less to the fluorescence signal increase. Things look somewhat different for the $\mathrm{F}_{\mathrm{g}}=1 \rightarrow \mathrm{F}_{\mathrm{e}}=1$ transition, in which the ratio of the contrast to the dark-state population experiences a maximum when the $h f_{s}$ split is roughly equal to the Doppler width, and the part to the left of the maximum is suppressed by a neighbouring bright resonance $\left(F_{g}=1 \rightarrow F_{e}=2\right)$ due to Doppler's broadening; when the maximum is reached, similar decrease is observed as in the case with the other two dark resonances.

We will examine the asymptotic values of the ratio of magneto-optical resonance's contrast $v s$. population in the dark states reached at large $h f s$ splitting. Transitions (1) to (3) tend to the following values: $2.55,1.47$ and 1.73 ; normalizing to the largest value we obtain the ratio: 1:0.58:0.68. Some basic deduction can lead us to nearly the same ratio if we try to understand how the resonances are formed. 

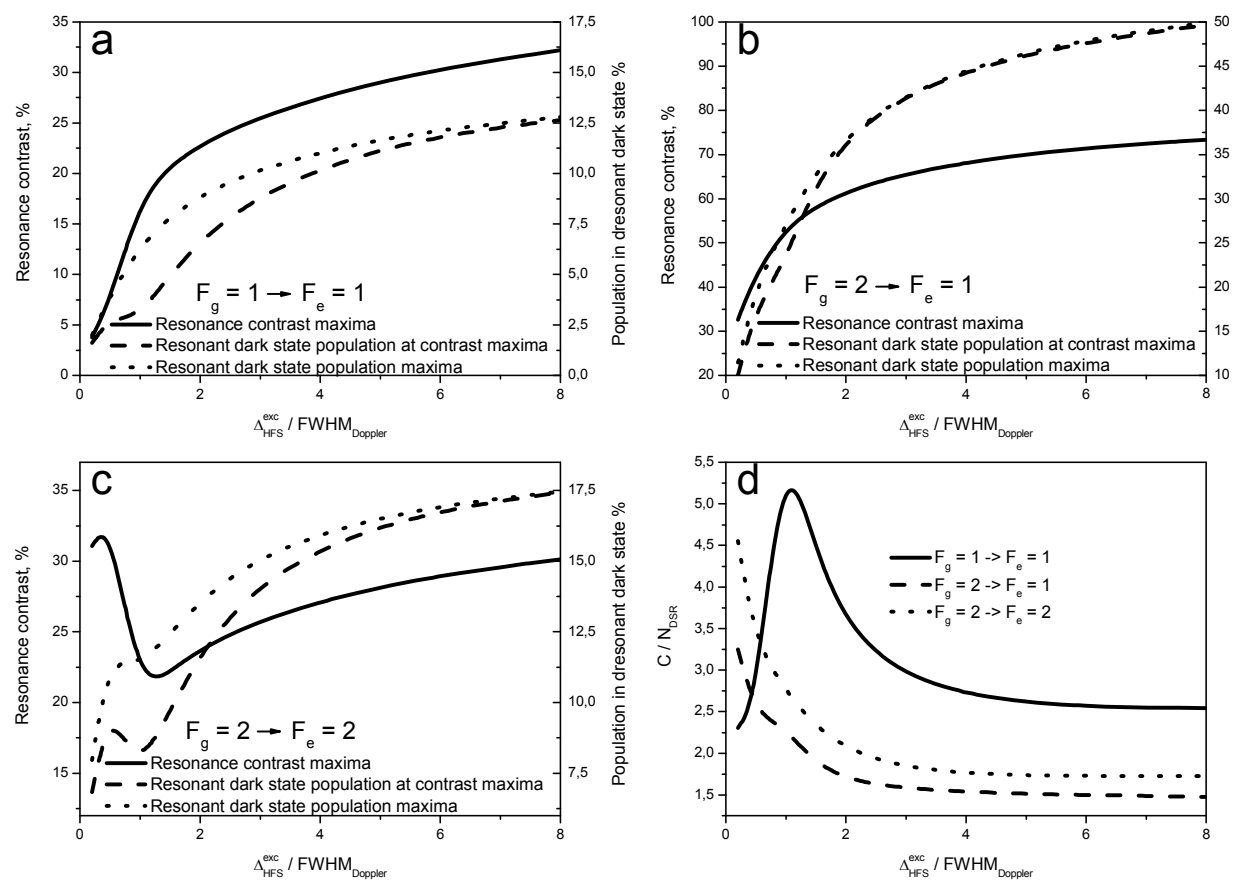

Fig. 7. $(a-c)$ : Maximum of dark resonance contrast (solid), population in resonant dark state at maximum contrast (dashed), and maximum of dark state population (dotted line) for given $h f s$ split, for hyperfine transitions $\mathrm{F}_{\mathrm{g}}=1 \rightarrow \mathrm{F}_{\mathrm{e}}=1(a), \mathrm{F}_{\mathrm{g}}=2 \rightarrow \mathrm{F}_{\mathrm{e}}=1(b)$ and $\mathrm{F}_{\mathrm{g}}=2 \rightarrow \mathrm{F}_{\mathrm{e}}=2(c)$; (d) maximum contrast to dark state population ratio.

We have to remember that the lines in Fig. $7 d$ represent the ratio of the contrast of the magneto-optical resonance $v s$. the fraction of the atoms trapped in the resonant dark state, which has a different level of degeneracy for different transitions; e.g., for transition (1) $\left(\mathrm{F}_{\mathrm{g}}=1 \rightarrow \mathrm{F}_{\mathrm{e}}=1\right)$ the resonant ground state has three magnetic sublevels (out of a total of 8 in the ground state), while for transitions (2) and (3) it has five magnetic sublevels. If we now look at the ratio of the total magnetic sublevels vs. the resonant ones, we would for all three transitions obtain the following values: $2.67: 1.6: 1.6$, which, when normalized, become 1:0.6:0.6; this is quite close to the one obtained from Fig. $7 d$. The slight deviations can be explained by the number of bright states in transitions that could be occupied by the atoms "released" from the dark states by the magnetic field (destruction of bright states by magnetic field is some three times slower because of the smaller Landé factor in the excited state). It turns out that transition (3) has more bright states, thus leading to a higher dark resonance contrast for the same dark state population. Finally, it should be noted that mutual ratios of contrast to dark-state population have roughly the same values for all $h f s$ splits shown in Fig. $7 d$, also in the region where these are rapidly changing at small splitting, except, of course, the region for transition (1) that is influenced by the bright resonance.

\section{CONCLUSIONS}

The results of theoretical simulations for dark magneto-optical resonance formation on the alkali $D_{l}$ line have been presented considering a fictional atomic structure for which the hyperfine constant for the excited state can be altered at 
will. It has been observed that the maxima of dark resonance contrast are driven towards larger laser power densities if the hyperfine splitting is increased; this, in turn, means that the ratio between the observed resonance contrast and the fraction of atoms trapped in the so-called dark state decreases because optical pumping of atoms away from the resonant hyperfine state becomes more effective. Another vital observation is that for all possible dark state configurations of the $D_{l}$ line maxima of the dark state population, if the $h f s$ splitting is small, are reached at higher Rabi frequencies (and thus higher laser power densities) than the maxima in the optically observable resonance contrast. For the most explicit dark resonance configuration $\left(\mathrm{F}_{\mathrm{g}}>\mathrm{F}_{\mathrm{e}}\right)$ this situation is inversed at larger hyperfine splitting. It is interesting to note that the maxima of resonance contrast and the dark state population coincide for the $h f_{s}$ splitting value that is roughly equal to that in the Caesium $D_{l}$ line. Thus, the most effective laser power density to trap atoms in the dark state can be simply detected by finding the largest value of dark resonance contrast. Finally, the ratio between the dark resonance contrast and the number of atoms trapped in the dark state has been explored; it is shown that this ratio in various dark resonance configurations can easily be explained if we consider the number of degenerate atomic states responsible for the dark-state formation.

\section{ACKNOWLEDGEMENTS}

The author acknowledges the valuable time spent in the research group led by Professors M. Auzinsh and R. Ferber at the Laser Centre of the University of Latvia. The author is grateful for the support from the ESF project Nr. 2009/0138/1DP/ 1.1.2.1.2./09/IPIA/VIAA/004.

\section{REFERENCES}

1. Nagel, A., Graf, L., Naumov, A., Mariotti, E., Biancalana, V., Meschede, D., \& Wynands, R. (1998). Experimental realization of coherent dark-state magnetometers. Europhys. Lett., 44 (1), 31; DOI: 10.1209/epl/1998-00430-0

2. Aspect, A., Arimondo, E., Kaiser, R., Vansteenkiste, N., \& Cohen-Tannoudji, C. (1988). Laser cooling below the one-photon recoil energy by velocity-selective coherent population trapping. Phys. Rev. Lett., 61, 826-829; DOI: 10.1103/PhysRevLett.61.826

3. Kitching, J., Hollberg, L., Knappe, S., \& Wynands, R. (2001). Compact atomic clock based on coherent population trapping. Electron. Lett.,37(24), 1449-1551.

4. Hau, L.V., Harris, S.E., Dutton, Z., \& Behroozi, C.H. (1999) Light speed reduction to 17 metres per second in an ultracold atomic gas. Nature, 397, 594-598; DOI: $10.1038 / 17561$

5. Schmieder, R.W., Lurio, A., Happer, W., \& Khadjavi, A. (1970). Level-crossing measurement of lifetime and $h f s$ constants of the ${ }^{2} \mathrm{P}_{3 / 2}$ states of the stable alkali atoms Phys. Rev. A 2 1216-1228 DOI: 10.1103/PhysRevA.2.1216

6. Alzetta, G., Gozzini, A., Moi, L. \& Orriols, G. (1976). An experimental method for the observation of r.f. transitions and laser beat resonances in oriented Na vapour. Il Nuovo Cimento B 36 (1) 5-20 DOI: 10.1007/BF02749417

7. Dancheva, Y., Alzetta, G., Cartalava, S., Taslakov, M., \& Andreeva, C. (2000). Coherent effects on the Zeeman sublevels of hyperfine states in optical pumping of Rb by monomode diode laser. Optics Communications, 178 (1-3), 103-110. DOI: 10.1016/S0030-4018(00)00643-X

8. Auzinsh, M., Ferber, R., Gahbauer F., Jarmola, A., \& Kalvans, L. (2009). Nonlinear 
magneto-optical resonances at $\mathrm{D}_{1}$ excitation of ${ }^{85} \mathrm{Rb}$ and ${ }^{87} \mathrm{Rb}$ for partially resolved hyperfine $\mathrm{F}$ levels. Phys. Rev. A, 79, 053404-053412. DOI: 10.1103/PhysRevA.79.053404.

9. Andreeva, C., Cartaleva, S., \& Dancheva, Y. (2002). Coherent spectroscopy of degenerate two-level systems in Cs. Phys. Rev. A, 66, 012502-012513, DOI: 10.1103/PhysRevA.66.012502.

10. Gateva, S., Alipieva, E., \& Taskova, E. (2005). Power dependence of the coherentpopulation-trapping resonances registered in fluorescence and transmission: Resonance-width narrowing effects, Phys. Rev. A 72 025805-025809 DOI: 10.1103/PhysRevA.72.025805

11. Stenholm, S. (2005). Foundations of Laser Spectroscopy. Minneola, New York: Dover Publications, Inc.

12. Allen, A., \& Eberly, J.H. (1975). Optical Resonance and Two Level Atoms. New York: Wiley.

13. Auzinsh, M., Ferber, R., Gahbauer F., Jarmola, A., \& Kalvans, L. (2008). F-resolved magneto-optical resonances in the $\mathrm{D}_{1}$ excitation of Caesium: Experiment and theory. Phys. Rev. A, 78, 013417-013424. DOI: 10.1103/PhysRevA.78.013417

14. Auzinsh, M., Ferber, R., Gahbauer F., Jarmola, A., Kalvans, L., Papoyan, A., \& Sarkisyan, D. (2010). Nonlinear magneto-optical resonances at $\mathrm{D}_{1}$ excitation of ${ }^{85} \mathrm{Rb}$ and ${ }^{87} \mathrm{Rb}$ in an extremely thin cell. Phys. Rev. A, 81, 033408-033417. DOI: 10.1103/PhysRevA.81.033408

\section{PIESĀTINĀŠSANĀS EFEKTI TUMŠAJĀS MAGNETO-OPTISKAJĀS REZONANSĒS, KAS UZN̦EMTAS UZ SĀRMU METĀLU $D_{l}$ LİNIJAS}

L. Kalvāns

\section{Kopsavilkums}

Rakstā aplūkoti teorētiski pētījumi par tumšās magneto-optiskās rezonanses piesātināšanos, ja to novēro uz sārmu metālu $D_{l}$ spektrālās līnijas. Izmantojot uz optiskajiem Bloha vienādojumiem balstītu teorētisko modeli, veiktas skaitliskas simulācijas sārmu metālu $D_{l}$ ierosmei. Tiek meklēts lāzera jaudas blīvums, pie kura tumšās rezonanses kontrasts sasniedz maksimālo vērtību un šie rezultāti korelēti ar lāzera inducētās kvantu superpozīcijas stāvokḷu apdzīvotību. Lai modelētu dažādu supersīkstruktūras pāreju ierosināšanas efektivitāti, skaitliskajā simulācijas tiek veiktas iedomātam atomam, kuram iespējams mainīt supersīkstruktūras konstanti. 